\title{
A Sub-pc-scale Acceleration of the Radio Jet of NGC 6251
}

\author{
Hiroshi Sudou, Yoshiaki Taniguchi, and Osamu Kaburaki \\ Astronomical Institute, Graduate School of Science, Tohoku University, \\ Aoba, Sendai, Miyagi, Japan \\ Youichi Ohyama, Seiji Kameno, Satoko Sawada-Satoh, Makoto Inoue, \\ and Tetsuo Sasao \\ National Astronomical Observatory, 2-21-1 Osawa, Mitaka, Tokyo, \\ Japan
}

\begin{abstract}
In order to investigate the genesis of powerful radio jet, we have mapped the central $10 \mathrm{pc}$ region of the nearby radio galaxy NGC 6251 with a $0.2 \mathrm{pc}$ resolution using VLBI at two radio frequencies, $5 \mathrm{GHz}$ and $15 \mathrm{GHz}$, we have found the sub-parsec-scale counterjet for the first time in this radio galaxy. This discovery allows us to investigate the jet acceleration based on the relativistic beaming model.
\end{abstract}

\section{Introduction}

NGC 6251 is one of apparently brightest powerful radio galaxies in the nearby universe and thus has been investigated extensively using VLBI (e.g., Jones et al. 1986). So far these measurements failed to detect the pc-scale counterjet. If we could detect it, it will be possible to give many important observational constraints on the jet geometry and then the physical process of jet acceleration. In order to find evidence for such sub-pc-scale counterjet in NGC 6251, we have performed new high-resolution VLBI observations using HALCA (Hirabayashi et al. 1998).

\section{Observations and Results}

NGC 6251 was observed at $5 \mathrm{GHz}$ using VSOP on 30 April 1998 and at $15 \mathrm{GHz}$ using VLBA on 2 June 1998. In order to perform beam-size-matched comparison between $5 \mathrm{GHz}$ and $15 \mathrm{GHz}$, we restored the two images with a same spatial resolution of $0.50 \times 0.50$ mas (Figure 1 ).

Careful comparison between the two maps at 5 and $15 \mathrm{GHz}$ reveals that the angular separation between the first and second brightest peak is different between the two maps, i.e., the separation at $15 \mathrm{GHz}$ is larger by 0.3 mas $(0.14$ pc) than that at $5 \mathrm{GHz}$. By comparing the detailed jet structures between the two maps (i.e., the wiggle pattern and the intensity profile), we attribute this difference to the apparent positional difference of the brightest peak, rather than 

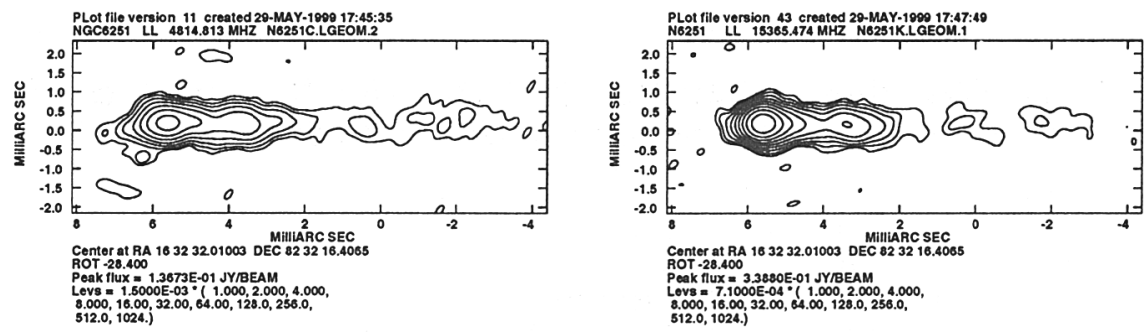

Figure 1. The images of NGC 6251 at $5 \mathrm{GHz}$ (left) and at $15 \mathrm{GHz}$ (right). Both images are rotated clockwise on the sky by $28^{\circ} .4$.

the different position of the knot because it is likely that the core at $5 \mathrm{GHz}$ suffers from strong absorption by free-free absorption.

We found a faint component which is extended to the opposite side of the main jet. Since this component has a steep spectral index, it is strongly suggested that this is the sub-pc-scale counterjet. Using the jet/counterjet intensity ratio, $R$, together with the Doppler beaming model, we estimated the jet velocity. Since $R$ increases with increasing distance from the core, it is suggested that the jet is accelerated at sub-pc-scale region. Assuming that the viewing angle of the jet is $30^{\circ}$, we show that the jet is accelerated from $\approx 0.13 c$ at $0.5 \mathrm{pc}$ to $\approx 0.42 c$ at $1.0 \mathrm{pc}$.

Acknowledgments. HS is grateful to the IAU organizing committee for travel support. The National Radio Astronomy Observatory is a facility of the National Science Foundation, operated under a cooperative agreement by Associated Universities, Inc.

\section{References}

Jones, D. L. et al. 1986, ApJ, 305, 684

Hirabayashi, H. et al. 1998, Science, 281, 1825 